\title{
Differential effects of scopolamine and lorazepam on working memory maintenance versus manipulation processes
}

\author{
Miriam Z. Mintzer and Roland R. Griffiths \\ Johns Hopkins University, Baltimore, Maryland
}

\begin{abstract}
Between-study comparisons of benzodiazepine and anticholinergic drugs on working memory suggest that anticholinergics may produce greater impairment in maintenance processes, whereas benzodiazepines may produce greater impairment in manipulation processes. This study directly compared acute effects of the benzodiazepine lorazepam ( 1.0 and $2.0 \mathrm{mg} / 70 \mathrm{~kg}$, orally administered) and the anticholinergic scopolamine $(0.25 \mathrm{and} 0.50 \mathrm{mg} /$ $70 \mathrm{~kg}$, subcutaneously administered) on working memory maintenance (storage and rehearsal) and manipulation processes in a placebo-controlled, double-dummy, double-blind, crossover design in 20 healthy volunteers. Using a modified Sternberg paradigm, storage, rehearsal, and manipulation processes were parametrically manipulated by varying memory load, delay between stimulus presentation and test, and number of operations performed on the letter strings, respectively, while controlling for drug effects on nonmemory processes. As predicted, the results suggested greater impairment in maintenance processes (rehearsal) with scopolamine than with lorazepam and greater impairment in manipulation processes with lorazepam than with scopolamine. In addition, the results suggested greater overall slowing of working memory processes with lorazepam.
\end{abstract}

It is well established that benzodiazepine (e.g., diazepam, or Valium; lorazepam, or Ativan) and anticholinergic (e.g., scopolamine) drugs induce temporary amnesia when administered acutely to healthy volunteers (for reviews, see Curran, 1991, 2000; Kopelman, 1986; Polster, 1993). Several researchers (Duka, Curran, Rusted, \& Weingartner, 1996; Hirshman, Passannante, \& Arndt, 2001; Mintzer \& Griffiths, 2001b; Polster, 1993; Reder et al., 2006) have argued that, like neuropsychological studies of brain-damaged patients, which have played a critical role in advancing the understanding of normal and abnormal memory mechanisms, investigation of drug-induced amnesia can also be a powerful tool for elucidating memory mechanisms. In fact, investigation of drug-induced amnesia has several distinct advantages over traditional studies of amnesic patients. Most importantly, unlike the memory deficits found in amnesic patients, effects of drugs on memory processes are reversible and can be empirically manipulated in controlled laboratory experiments with large numbers of healthy volunteers. Furthermore, the quantitative dosing properties of drugs can be exploited to produce a graded amnesic effect in a dose-effect design. Findings of selective effects of drugs on particular aspects of memory performance but not others, or of dissociations between drugs, can provide converging evidence with data from nonpharmacological studies for the dissociability of specific processes or subcomponents (see Hirshman, Fisher, Henthorn, Arndt, \& Passannante, 2002; Hirshman et al., 2001; Mintzer, 2003; Mintzer \& Griffiths, 2001b). In addition, comparison of memory-impairing drugs with distinct neurochemical or pharmacological mechanisms of action can elucidate the brain mechanisms underlying specific cognitive processes.

The present experiment was designed to compare the acute effects of the benzodiazepine lorazepam and the anticholinergic scopolamine on working memory processes. Benzodiazepines facilitate the action of gammaaminobutyric acid (GABA) by acting as agonists at specific sites on the $\mathrm{GABA}_{\mathrm{A}}$ receptor complex (Mohler \& Okada, 1977; Squires \& Braestrup, 1977), whereas scopolamine inhibits the action of acetylcholine by acting as an antagonist at muscarinic cholinergic receptors (Ketchum, Sidell, Crowell, Aghajanian, \& Haines, 1973). Working memory refers to the temporary maintenance and online manipulation of a limited amount of information in the service of current behavioral goals (Baddeley, 1986, 1992; for recent reviews, see D'Esposito, 2001; Smith \& Jonides, 1998). The concept of working memory was originally introduced by Baddeley (1986) and replaced earlier conceptualizations of a unitary, passive, short-term storage system (Atkinson \& Shiffrin, 1968). In Baddeley's model, working memory is a multicomponent system consisting of a number of limited-capacity (Miller, 1956), material-specific "slave systems" (e.g., the phonological loop for verbal material and the visuospatial sketchpad for visual material) involved in temporarily maintaining 
information not currently available in the environment, and a central executive (or set of executive processes) involved in controlling manipulations (e.g., reorganization, updating) performed on that information. Evidence that the maintenance and manipulation components of working memory are separate and dissociable, with distinct neural substrates, comes from animal single cell recordings, neuropsychological studies of brain-damaged patients, and cognitive psychopharmacological and functional neuroimaging studies in healthy volunteers (Barde \& Thompson-Schill, 2002; D'Esposito, Postle, \& Rypma, 2000; Glahn et al., 2002; Honey et al., 2003; Kim, Glahn, Nuechterlein, \& Cannon, 2004; Petrides, 1994). There is also neuropsychological and neuroimaging evidence that the maintenance component consists of dissociable subcomponents: a passive storage buffer (e.g., the phonological store for verbal material), and an active rehearsal mechanism for refreshing and keeping active the contents of the buffer (e.g., an articulatory control process for verbal material; see D'Esposito \& Postle, 1999; Smith \& Jonides, 1998).

Research on the neurochemical modulation of working memory suggests that working memory is modulated by several different neurotransmitter systems, and that the dopaminergic and cholinergic systems play particularly important roles (for a review, see Barch, 2004). As described in detail below, the present comparison between lorazepam and scopolamine was motivated by evidence from both nonhuman animal and human studies that benzodiazepines and anticholinergics may differ in their effects on specific working memory processes. Both benzodiazepines and scopolamine have been shown to impair performance on working memory tasks (e.g., delayed matching-to-sample tasks) in both rats and nonhuman primates (Chrobak \& Napier, 1992; Hironaka, Miyata, \& Ando, 1992; Ingles, Beninger, Jhamandas, \& Boegman, 1993; White, Simson, \& Best, 1997). Additional support for the involvement of the cholinergic system, in particular, in working memory, comes from findings that the acetylcholinestrase inhibitor physostigmine enhances working memory performance in animals, healthy adult volunteers, and patients with Alzheimer's disease (Furey, Pietrini, Alexander, Schapiro, \& Horwitz, 2000; Furey, Pietrini, \& Haxby, 2000; Furey et al., 1997; Glasky, Melchior, Pirzadeh, Heydari, \& Ritzmann, 1994; Levy et al., 1994; Sano et al., 1993; Terry, Jackson, \& Buccafusco, 1993).

For both benzodiazepines and scopolamine, results of previous studies that included short-term or working memory tasks suggest that acute drug administration does not impair performance on simple span tasks (e.g., the digit span task, in which participants are required to repeat a random sequence of digits immediately after their presentation; Wechsler, 1981), but may impair performance on more complex tasks, such as logical reasoning and planning (Bishop, Curran, \& Lader, 1996; Coull, Middleton, Robbins, \& Sahakian, 1995; Rusted, 1988; Rusted, Eaton-Williams, \& Warburton, 1991; Rusted \& Warburton, 1988). However, few studies have explicitly manipulated experimental conditions to allow conclusions to be drawn about drug effects or differences between drugs in effects on specific working memory processes. One notable exception is a study by Robbins et al. (1997) that examined dose effects of the benzodiazepine diazepam $(5,10 \mathrm{mg})$ and scopolamine $(200,400,600 \mu \mathrm{g})$ in separate placebo-controlled experiments using identical measures. In a delayed matching-to-sample task, participants were presented with a complex visual pattern for a fixed amount of time, and after the pattern disappeared, they were asked to identify the previously presented pattern from among three distractor patterns; the delay $(0,4,12 \mathrm{sec})$ between pattern presentation and identification was manipulated. The results suggested that scopolamine impaired identification performance in a delay-dependent manner (i.e., increased impairment with increasing delay), whereas diazepam impaired performance in a delay-independent manner (although conclusions are somewhat limited by the near-ceiling level of accuracy in the placebo condition at all three delays in the diazepam experiment and the fact that scopolamine and diazepam were not directly compared in a single experiment). Given that memory load was not manipulated (i.e., each trial involved presentation of only one pattern), presumably storage requirements were held constant and only rehearsal requirements changed as a function of delay; thus, results suggest that scopolamine impairs both the storage and rehearsal components of working memory, whereas diazepam impairs only storage. A similar pattern of results was found in a comparison of effects of diazepam and scopolamine on a delayed matching-to-sample task in rhesus monkeys (Hironaka et al., 1992): Scopolamine produced delay-dependent impairment, whereas diazepam produced delay-independent impairment.

Although the task used in Robbins et al. (1997; and in Hironaka et al., 1992) required that material be maintained over a particular duration, it did not require that any manipulations be performed on the material. Interestingly, results of a study recently conducted in our laboratory with lorazepam and scopolamine suggest that when maintenance requirements are relatively low but manipulation is required, the opposite pattern of results is observed (Mintzer \& Griffiths, 2003). Specifically, in the two-back task that requires participants to temporarily maintain in memory and continuously update the identity and order of the two previous letters in a sequence of letters presented consecutively on the screen, more impairment was observed under lorazepam than under scopolamine conditions. The possibility of differential effects of the two drugs on maintenance versus manipulation processes is intriguing. However, conclusions based on between-study comparisons are inherently limited. Therefore, the present study was designed to directly compare the acute dose effects of lorazepam $(1.0$ and $2.0 \mathrm{mg} / 70 \mathrm{~kg}$, orally administered) and scopolamine (0.25 and $0.50 \mathrm{mg} / 70 \mathrm{~kg}$, subcutaneously administered) on working memory maintenance (storage and rehearsal) and manipulation processes in a placebo-controlled, double-dummy, double-blind, crossover design in healthy volunteers. To facilitate direct comparison of lorazepam and scopolamine at corresponding dose levels, doses of the two drugs were selected to 
produce comparable decrements in psychomotor performance on the basis of previous studies. Using a variant of the Sternberg delayed response paradigm, storage, rehearsal, and manipulation processes were parametrically manipulated by varying the memory load, the delay between stimulus presentation and test, and the number of operations performed on the letter strings, respectively; a nonmemory condition was included to control for drug effects on perceptual/attentional processes. Based on the results of Robbins et al. (1997) and Mintzer and Griffiths (2003), it was hypothesized that the effects of memory load would be similar for scopolamine and lorazepam, the effects of delay would be relatively greater for scopolamine, and the effects of number of operations would be relatively greater for lorazepam.

\section{METHOD}

\section{Participants}

Participants were recruited from local colleges and from the community-at-large in Baltimore, MD. Twenty adult volunteers ( 9 male) completed the experiment. The participants ranged in age from 19 to 43 years $(M=27)$ and in weight from 49 to $91 \mathrm{~kg}(M=$ $70)$, and reported having completed 12 to 20 years of education $(M=15)$. None of the participants reported histories of drug or alcohol abuse. The participants were requested to refrain from using all psychoactive drugs. Each session, before drug administration, the participants were tested for the presence of various drugs in urine (benzodiazepines, opioids, methadone, and cocaine) using an EMIT system (Syva Co., Palo Alto, CA) and the presence of alcohol in expired air using a Breathalyzer test. All of the participants were in good health (as determined by a medical history and personal interview), with no contraindications to benzodiazepine or anticholinergic drugs. Individuals with current or past histories of psychiatric disorders were excluded. In the female participants, urine pregnancy tests conducted prior to the first session were negative. The study was approved by the Institutional Review Board of Johns Hopkins University School of Medicine. The participants gave their informed, written consent before beginning the study, and were paid for their participation.

\section{General Procedure}

The participants completed a total of five sessions as outpatients at the Behavioral Pharmacology Research Unit of Johns Hopkins University School of Medicine. Successive sessions were separated by a minimum of $48 \mathrm{~h}$. Single doses of placebo, $1.0 \mathrm{mg} / 70 \mathrm{~kg}$ lorazepam, $2.0 \mathrm{mg} / 70 \mathrm{~kg}$ lorazepam, $0.25 \mathrm{mg} / 70 \mathrm{~kg}$ scopolamine, and $0.50 \mathrm{mg} / 70 \mathrm{~kg}$ scopolamine were administered across the five sessions in a double-blind (i.e., both the participant and the staff member directly involved in data collection were blind to specific drug conditions in order to minimize the confounding of data by expectations about the nature of drug effects), within-subjects crossover design. Lorazepam was administered orally and scopolamine was administered subcutaneously, as in previous studies, due to its more variable absorption rate after oral administration (Bishop et al., 1996; Mintzer \& Griffiths, 2001a, 2003; Wesnes, Simpson, \& Kidd, 1988). Given that scopolamine and lorazepam were administered by different routes, in order to further mask the drug condition, drug administration was "double dummy," such that participants received both an oral dose (of either lorazepam or lactose placebo capsules ingested with approximately $150 \mathrm{ml}$ of water) and a subcutaneous injection (of either scopolamine or saline placebo; administered in a constant volume of $1 \mathrm{ml}$ in the upper right arm) during each session. In order to synchronize the peak effects of lorazepam and scopolamine on the basis of pharmacokinetic and behavioral data, a staggered dosing regimen was used, in which the oral dose was ad- ministered $1 \mathrm{~h}$ earlier than the subcutaneous dose. The order of drug conditions was determined by two Latin squares, using the Williams method (Williams, 1949), to achieve balance in presentation order and in the order of drug conditions relative to one another.

Lorazepam doses were prepared from commercially available $2-\mathrm{mg}$ tablets (Wyeth Laboratories, Philadelphia, PA). The tablets were crushed, and the doses were adjusted for participant body weight and dispensed in size 0 opaque capsules. Scopolamine doses (expressed as salt) were prepared from scopolamine hydrobromide trihydrate, USP (Spectrum Pharmacy Products, Gardena, CA); the doses were adjusted for participant body weight and dissolved in saline to a volume of $1 \mathrm{ml}$. The primary dependent measures involved the working memory tasks. In addition, two psychomotor performance tasks (circular lights and balance) were administered at regular intervals during each session to verify the comparability of the selected doses of lorazepam and scopolamine. In the circular lights task, the participants pressed a series of 16 buttons as rapidly as possible in response to the randomly sequenced illumination of their associated lights, and in the balance task, the participants were asked to stand upright on one foot with their eyes closed and their arms extended to the side, at shoulder height, for a maximum of $30 \mathrm{sec}$ on each foot (Mintzer, Frey, Yingling, \& Griffiths, 1997). It was expected that lorazepam and scopolamine would produce similar decrements on these tasks at corresponding dose levels (i.e., low, $1.0 \mathrm{mg} / 70 \mathrm{~kg}$ lorazepam, $0.25 \mathrm{mg} / 70 \mathrm{~kg}$ scopolamine; high, $2.0 \mathrm{mg} / 70 \mathrm{~kg}$ lorazepam, $0.50 \mathrm{mg} / 70 \mathrm{~kg}$ scopolamine). Additional assessments were also administered at regular intervals, but those data will not be reported here.

\section{Working Memory Tasks: Maintenance and Manipulation}

The two working memory tasks were variants of the classic Sternberg task (Sternberg, 1969) and used procedures similar to those described by Postle, Berger, and D'Esposito (1999) and D'Esposito, Postle, Ballard, and Lease (1999). They were administered on an Apple Macintosh microcomputer. The tasks began 110 min after capsule administration (i.e., 55 min after subcutaneous injection) so that both tasks would be conducted within the period of peak effects for lorazepam and scopolamine. The order of the two tasks (maintenance and manipulation) was counterbalanced across participants, with a 5-min rest period between tasks. To stabilize performance prior to the experimental sessions, and to minimize variability across sessions, during the initial screening session, participants performed abbreviated versions of each task until they reached a criterial level of performance. During each experimental session, standardized instructions were read to the participants prior to each block of each task, and practice trials were presented prior to the experimental trials to ensure that the participants understood and performed the tasks correctly.

Maintenance task. A memory set consisting of randomly selected and randomly ordered consonant letters (excluding $\mathrm{L}$ and $\mathrm{Y}$; e.g., PCZM) was presented on the screen followed by a probe consisting of a letter-digit pair (e.g., c-2), and participants were asked to decide whether the probed letter had appeared in the memory set in the ordinal position represented by the digit (e.g., $2=2$ nd position in the memory set). The probed letter was always a letter that had appeared in the memory set. To prevent simple perceptual matching to the memory set stimuli (which were presented in uppercase), the probe was always presented in lowercase. Effects on storage processes were tested by parametrically varying the size of the memory set $(5,7$ letters), and effects on rehearsal processes were tested by parametrically varying the delay between memory set presentation and testing (i.e., probe presentation; $0,4,12 \mathrm{sec}$ ). The participants completed two blocks, one in the 5-letter condition and one in the 7-letter condition. The order of the two blocks was counterbalanced across participants. Each block consisted of 12 trials in each of the three delay conditions and 12 trials in a control condition in which the memory set remained on the screen during probe presentation (i.e., 48 trials total per block). Within a block, trials in all of the conditions were randomly intermixed. The control condition 
was designed to control for drug effects on nonmemory processes (e.g., motor coordination, perception, attention, and other cognitive processes). Within each condition, the probed digit represented the correct position of the probed letter on half of the trials.

The memory set appeared on the screen for $4 \mathrm{sec}$, followed by the predetermined delay and the presentation of the probe for a period of $7 \mathrm{sec}$, during which the participant responded by using the computer mouse to click on a button labeled yes or no. Reaction time (RT) from the onset of the probe was recorded. After each response, the participants were required to return the mouse cursor to a screen position that was equidistant between the two response buttons; this position was indicated by a circle on the screen that was illuminated when the cursor touched it. There was a 2-sec intertrial interval (ITI).

Manipulation task. This task used stimuli and procedures similar to those described for the maintenance task, except that the participants were asked to perform manipulations on the stimuli in the memory set. Effects on manipulation processes were tested by parametrically varying the number of separate sequencing steps performed (0, 1, 2; see Berman, Austin-Lane, Esposito, Van Horn, $\&$ Weinberger, 1996). In the 0-manipulation condition, the participants were asked to make a decision about the ordinal position of the probed letter in the memory set, as described for the maintenance task. This condition required only maintenance processes. In the 1-manipulation condition, the participants were asked to alphabetize the letters, and to decide whether the probed letter would be in the ordinal position represented by the probed digit in the reordered string (e.g., for the memory set PCZM, the correct reordered string would be CMPZ). In the 2-manipulation condition, the participants were asked first to alphabetize the letters, and second, to move the final letter in the alphabetized string to the beginning of the alphabetized string, and to decide whether the probed letter would be in the ordinal position represented by the probed digit in the final reordered string (e.g., for PCZM, the correct reordered string would be ZCMP). The stimulus materials were identical to those in the maintenance task except that in addition to excluding the letters $\mathrm{L}$ and $\mathrm{Y}$, letters from the beginning $(\mathrm{B}, \mathrm{C}, \mathrm{D}, \mathrm{F})$ and end $(\mathrm{V}, \mathrm{W}, \mathrm{X}, \mathrm{Z})$ of the alphabet were also excluded to reduce the variability among trials in ease of alphabetization. Maintenance requirements were held constant by using a consistent delay $(0 \mathrm{sec})$ and memory set size (four stimuli) across trials. To minimize confusion and to ensure that the participants performed the appropriate task on each trial, trials in the three manipulation conditions (12 in each condition) were presented in separate blocks, and the appropriate set of instructions remained on the screen throughout. The order of the three blocks was random for each participant. In each of the three blocks, 12 control trials were randomly intermixed, in which participants were asked to perform the same task as in the other trials in that block, but the memory set remained on the screen during probe presentation. Thus, there were a total of 24 trials in each block. Within each condition, each of the possible probe positions was probed an equal number of times (i.e., there were 3 trials in each of the 4 possible probe positions) and the probed digit represented the correct position of the probed letter on half of the trials.

As in the maintenance task, the memory set appeared on the screen for $4 \mathrm{sec}$ and the probe was presented for a period of $7 \mathrm{sec}$, during which the participant responded by using the computer mouse to click on a button labeled yes or no. The RT from the onset of the probe was recorded. After each response, the participants were required to return the mouse cursor to a screen position that was equidistant between the two response buttons; this position was indicated by a circle on the screen that was illuminated when the cursor touched it. There was a 2 -sec ITI.

\section{Data Analysis}

For the working memory tasks, dependent variables were the proportion of correct responses and mean RT. RT data were prepared for analysis for each participant in each condition by removing outliers greater than 3 standard deviations above the participant's mean in that condition. RTs to incorrect responses were excluded. The proportion correct and RT data were analyzed by repeated measures ANCOVA, as described in detail for each task in the Results section. On three occasions (one block of the manipulation task during one session for each of 3 participants), a participant performed the wrong task, and data from the affected conditions were excluded from analysis. Therefore, PROC MIXED (a procedure in SAS that models missing data) was used to analyze data from the manipulation task. Data from the psychomotor performance tasks were analyzed by repeated measures ANOVA, with drug condition (placebo and four active drug conditions) and time (before drug administration and 80 and 230 min after capsule administration) as factors. For all analyses, significant main effects were followed up with $t$ tests, and significant interactions were followed up with analyses of simple effects of each independent variable at each level of the other independent variable (Keppel, 1991); comparisons among drug conditions were limited to comparisons of each active drug condition versus placebo and of corresponding doses of lorazepam and scopolamine (i.e., high-dose lorazepam vs. high-dose scopolamine; low-dose lorazepam vs. low-dose scopolamine). For all statistical tests, $p<.05$ was considered significant.

\section{RESULTS}

\section{Psychomotor Performance}

The ANOVA revealed a significant interaction between drug condition and time [for circular lights, $F(8,152)=$ $7.77, M S_{\mathrm{e}}=25.98$; for balance, $F(8,152)=5.77, M S_{\mathrm{e}}=$ $95.36]$. Both lorazepam and scopolamine produced doseand time-related decrements in performance on the circular lights and balance tasks, although for balance, low-dose lorazepam did not reach significance relative to placebo (Figure 1). Simple effects tests indicated no significant differences between corresponding doses of lorazepam and scopolamine at either of the postdrug timepoints, supporting the comparability of the selected doses.

\section{Working Memory: Maintenance Task}

As can be seen in Table 1, the mean proportion correct was higher and the mean RT was lower in the control condition (i.e., trials in which the memory set remained on the screen during probe presentation) than in the memory conditions (i.e., 0-, 4-, 12-sec delay), confirming that the control trials operated as expected. Therefore, the control condition was used to control for drug effects on nonmemory processes, as planned, by conducting an ANCOVA on mean proportion correct and mean RT for the memory conditions, with the control condition as covariate, and drug condition, memory set size (5, 7 letters), and delay $(0,4,12 \mathrm{sec})$ as factors.

For mean proportion correct, the ANCOVA revealed significant main effects of drug condition $[F(4,76)=8.11]$, memory set size $[F(1,19)=49.64]$, and delay $[F(2,38)=$ 33.60], and a significant drug condition $\times$ delay interaction $[F(8,152)=3.26]$ (see Figure 2 for adjusted means). There was no significant interaction between drug condition and memory set size or between memory set size and delay (both $F_{\mathrm{s}}<1$ ). Replicating the effect of memory load on working memory accuracy, the mean proportion correct was significantly lower in the 7-letter condition $(M=$ $.78)$ than in the 5-letter condition $(M=.86)$ (the memory set size main effect). Simple effects tests on the drug con- 

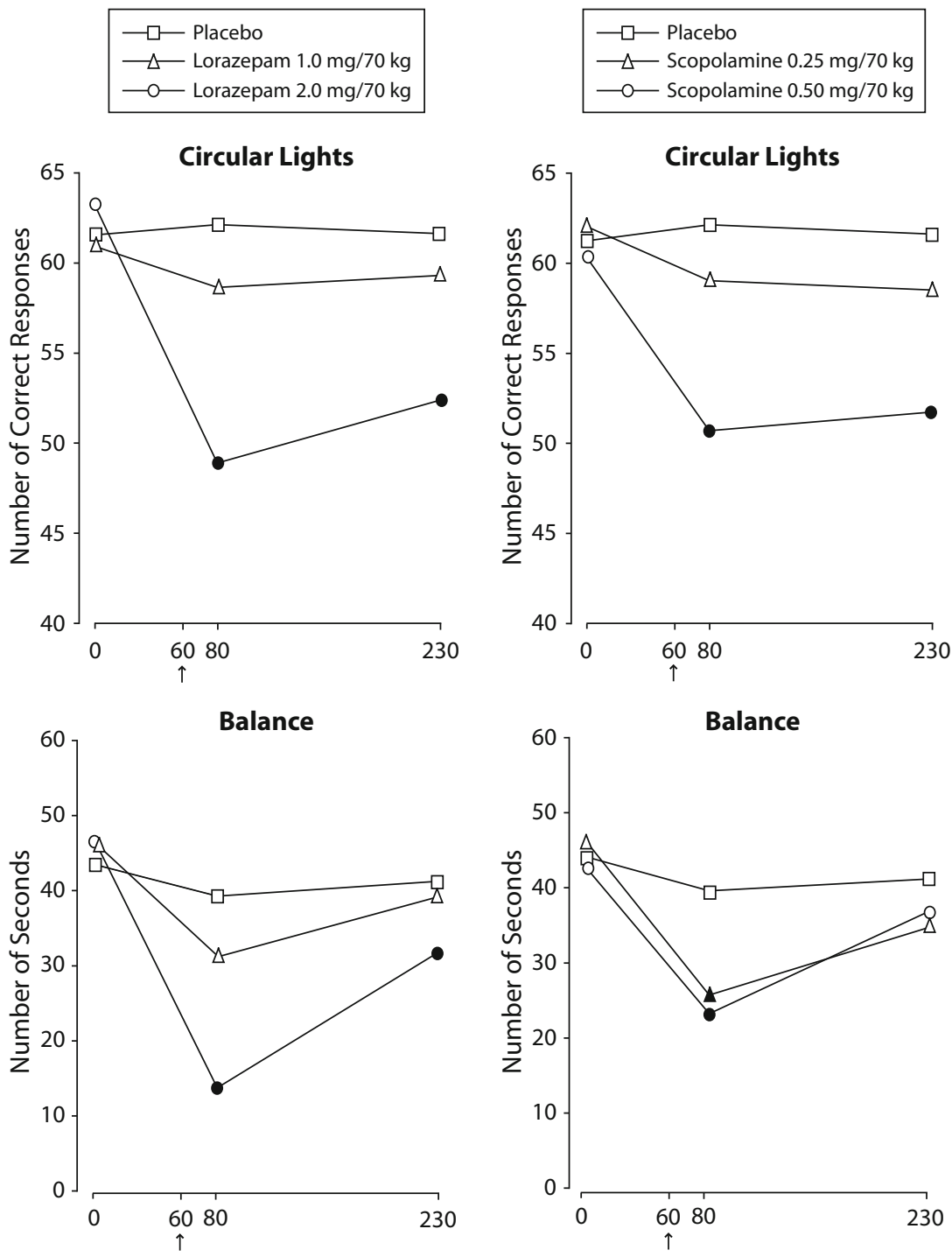

Time After Capsule Administration (min)

Time After Capsule Administration (min)

Figure 1. Lorazepam (left panel) and scopolamine (right panel) time-course functions for performance on circular lights (mean number of correct responses during a 60-sec trial) and balance (mean number of seconds balanced, out of a total of $60 \mathrm{sec}$ for both feet). $x$-axis: Time in minutes after capsule administration. The arrow indicates time of subcutaneous injection; 0 indicates predrug. A darkened symbol indicates an active drug value that is significantly different from the corresponding placebo value at the same timepoint.

dition $\times$ delay interaction revealed that the mean proportion correct was significantly lower in the high-dose lorazepam condition in comparison with placebo at all three delays, significantly lower in the high-dose scopolamine condition in comparison with placebo at the 4 - and 12 -sec delays, significantly lower in the low-dose lorazepam and low-dose scopolamine conditions in comparison with placebo at the 12-sec delay, and significantly lower in the high-dose scopolamine condition than in the high-dose lorazepam condition at the 4- and 12-sec delays.

For mean RT, the ANCOVA revealed significant main effects of drug condition $[F(4,76)=10.26]$, memory set size $[F(1,19)=30.80]$, and delay $[F(2,38)=25.68]$, but no significant interactions [for drug condition $\times$ memory set size, $F(4,76)=2.46$; for drug condition $\times$ delay, $F(8,152)=1.48$; for memory set size $\times$ delay, $F(2,38)=$ $0.34]$. Simple effects on the drug condition main effect revealed that the mean RT was significantly higher in the high-dose and low-dose lorazepam conditions in comparison with placebo, and significantly higher in the lowand high-dose lorazepam conditions in comparison with the corresponding scopolamine conditions (see Figure 3 for adjusted means by drug condition, collapsed across memory set size and delay because these factors did not interact significantly with drug condition). Replicating the effects of memory set size and delay on working mem- 
Table 1

Mean Proportions Correct and Reaction Times, With Standard Deviations, in the Maintenance Task As a Function of Drug Condition, Memory Set Size, and Delay

\begin{tabular}{|c|c|c|c|c|c|c|c|c|c|c|c|}
\hline & & & \multicolumn{4}{|c|}{ Lorazepam $(\mathrm{mg} / 70 \mathrm{~kg})$} & \multicolumn{4}{|c|}{ Scopolamine $(\mathrm{mg} / 70 \mathrm{~kg})$} \\
\hline & & \multicolumn{2}{|c|}{ Placebo } & \multicolumn{2}{|c|}{1.0} & \multicolumn{2}{|c|}{2.0} & \multicolumn{2}{|c|}{0.25} & \multicolumn{2}{|c|}{0.50} \\
\hline & & $M$ & $S D$ & $M$ & $S D$ & $M$ & $S D$ & $M$ & $S D$ & $M$ & $S D$ \\
\hline \multicolumn{12}{|c|}{ Proportion Correct } \\
\hline \multirow[t]{4}{*}{5 letters } & Control & .98 & .05 & .95 & .10 & .91 & .10 & .93 & .12 & .93 & .08 \\
\hline & $0 \mathrm{sec}$ & .96 & .05 & .90 & .14 & .85 & .14 & .87 & .12 & .82 & .18 \\
\hline & $4 \mathrm{sec}$ & .95 & .07 & .90 & .15 & .86 & .15 & .87 & .14 & .77 & .24 \\
\hline & $12 \mathrm{sec}$ & .93 & .10 & .80 & .21 & .78 & .19 & .83 & .14 & .73 & .20 \\
\hline \multirow{4}{*}{7 letters } & Control & .98 & .04 & .96 & .07 & .96 & .07 & .90 & .15 & .94 & .08 \\
\hline & $0 \mathrm{sec}$ & .88 & .10 & .83 & .13 & .79 & .16 & .79 & .18 & .83 & .16 \\
\hline & $4 \mathrm{sec}$ & .88 & .09 & .84 & .16 & .77 & .21 & .79 & .16 & .65 & .17 \\
\hline & $12 \mathrm{sec}$ & .85 & .13 & .76 & .15 & .73 & .21 & .69 & .20 & .62 & .19 \\
\hline \multicolumn{12}{|c|}{ Reaction Time (msec) } \\
\hline \multirow[t]{4}{*}{5 letters } & Control & 1,992 & 443 & 2,416 & 800 & 2,592 & 497 & 2,150 & 506 & 2,351 & 444 \\
\hline & $0 \mathrm{sec}$ & 2,218 & 439 & 2,580 & 500 & 2,951 & 488 & 2,311 & 551 & 2,541 & 469 \\
\hline & $4 \mathrm{sec}$ & 2,302 & 514 & 2,707 & 707 & 3,307 & 751 & 2,526 & 634 & 2,869 & 786 \\
\hline & $12 \mathrm{sec}$ & 2,353 & 545 & 2,849 & 727 & 3,435 & 632 & 2,583 & 446 & 2,890 & 771 \\
\hline \multirow[t]{4}{*}{7 letters } & Control & 2,229 & 466 & 2,541 & 662 & 3,077 & 739 & 2,528 & 553 & 2,691 & 786 \\
\hline & $0 \mathrm{sec}$ & 2,658 & 452 & 3,115 & 573 & 3,297 & 632 & 2,689 & 450 & 2,786 & 543 \\
\hline & $4 \mathrm{sec}$ & 2,827 & 565 & 3,152 & 669 & 3,574 & 562 & 3,190 & 723 & 3,160 & 552 \\
\hline & $12 \mathrm{sec}$ & 2,924 & 686 & 3,405 & 635 & 3,713 & 630 & 2,924 & 602 & 3,002 & 668 \\
\hline
\end{tabular}

ory RT, mean RT was significantly higher in the 7-letter condition $(M=3,030 \mathrm{msec})$ than in the 5-letter condition $(M=2,759 \mathrm{msec})$, and significantly higher at the 4 -sec $(M=2,961 \mathrm{msec})$ and 12 -sec $(M=3,008 \mathrm{msec})$ delays than at the $0-\sec (M=2,715 \mathrm{msec})$ delay.

\section{Working Memory: Manipulation Task}

As can be seen in Table 2, performance was not better (i.e., the mean proportion correct was not higher and the RT was not lower) in the control condition (i.e., trials in which the memory set remained on the screen during probe presentation) in comparison with the memory conditions in the 1- and 2-manipulation conditions. To the contrary, the mean RT was actually higher in the control condition than in the memory condition in the $1-$ and 2-manipulation conditions. In retrospect, it makes intuitive sense that having the original memory set remain on the screen while the participant is being probed about
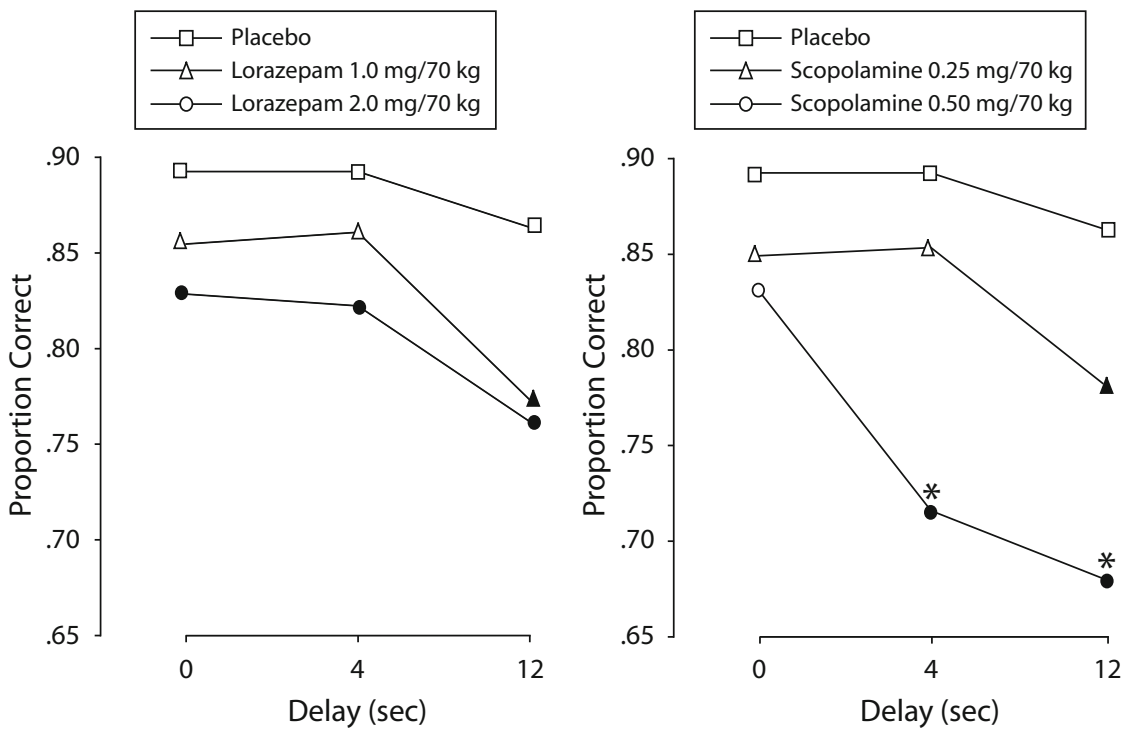

Figure 2. Mean proportion correct for the memory conditions of the maintenance task (adjusted means from the ANCOVA with the control condition as covariate; collapsed across memory set size) for lorazepam (left panel) and scopolamine (right panel) as a function of the delay between memory set presentation and probe presentation. A darkened symbol indicates an active drug value that is significantly different from the corresponding placebo value at the same delay. An asterisk (*) indicates a scopolamine value that is significantly different from the lorazepam value at the corresponding dose at the same delay. 


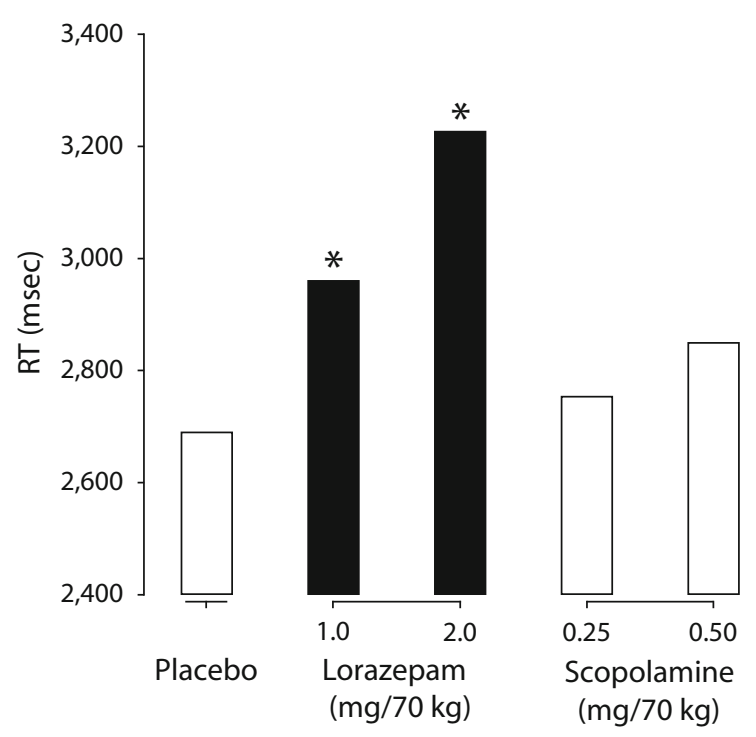

Figure 3. Mean reaction time for the memory conditions of the maintenance task (adjusted means from the ANCOVA with the control condition as covariate; collapsed across delay and memory set size) as a function of drug condition. A darkened bar indicates an active drug value that is significantly different from placebo. An asterisk $(*)$ indicates a lorazepam value that is significantly different from the scopolamine value at the corresponding dose.

a reordered string might be distracting, and that for the 1- and 2-manipulation conditions, the control condition actually does not serve as a nonmemory control, because the participant must still maintain the reordered string in working memory. Therefore, data from the control trials were excluded from the analyses and an ANCOVA was conducted on mean proportion correct and mean RT, including only the memory trials, with the 0-manipulation condition as covariate, and drug condition and number of manipulations $(1,2)$ as factors (to examine effects on ma- nipulation, while controlling for effects on maintenance). The adjusted means are shown in Figure 4 as a function of drug condition (collapsed across number of manipulations because there was no significant drug condition $\times$ number of manipulations interaction for proportion correct or RT; see below).

For mean proportion correct, the ANCOVA revealed a significant main effect of drug condition $[F(4,75)=$ $3.68]$, such that mean proportion correct was significantly lower in the high-dose lorazepam condition in comparison with placebo and high-dose scopolamine, and a significant main effect of number of manipulations $[F(1,19)=$ 18.72], such that mean proportion correct was significantly lower in the 2-manipulation condition $(M=.83)$ than in the 1-manipulation condition $(M=.89)$. There was no significant interaction between drug condition and number of manipulations $(F<1)$. For mean RT, the ANCOVA revealed a significant main effect of drug condition $[F(4,75)=8.71]$, such that mean RT was significantly higher in the high-dose lorazepam condition in comparison with placebo and high-dose scopolamine, and a significant main effect of number of manipulations $[F(1,19)=79.26]$, such that mean RT was significantly higher in the 2-manipulation condition $(M=3,099 \mathrm{msec})$ than in the 1-manipulation condition $(M=2,603 \mathrm{msec})$. There was no significant interaction between drug condition and number of manipulations $(F<1)$.

\section{DISCUSSION}

The pattern of results predicted on the basis of the between-study comparison of Robbins et al. (1997) and Mintzer and Griffiths (2003) was observed in the accuracy data. Specifically, the proportion correct was lower for high-dose scopolamine than for lorazepam at longer delays (4 and $12 \mathrm{sec}$; Figure 2), suggesting that scopolamine produces relatively greater impairment in rehearsal processes, whereas the proportion correct was lower for

Table 2

Mean Proportions Correct and Reaction Times, With Standard Deviations*, in the Manipulation Task As a Function of Drug Condition, Control Versus Memory, and Number of Manipulations

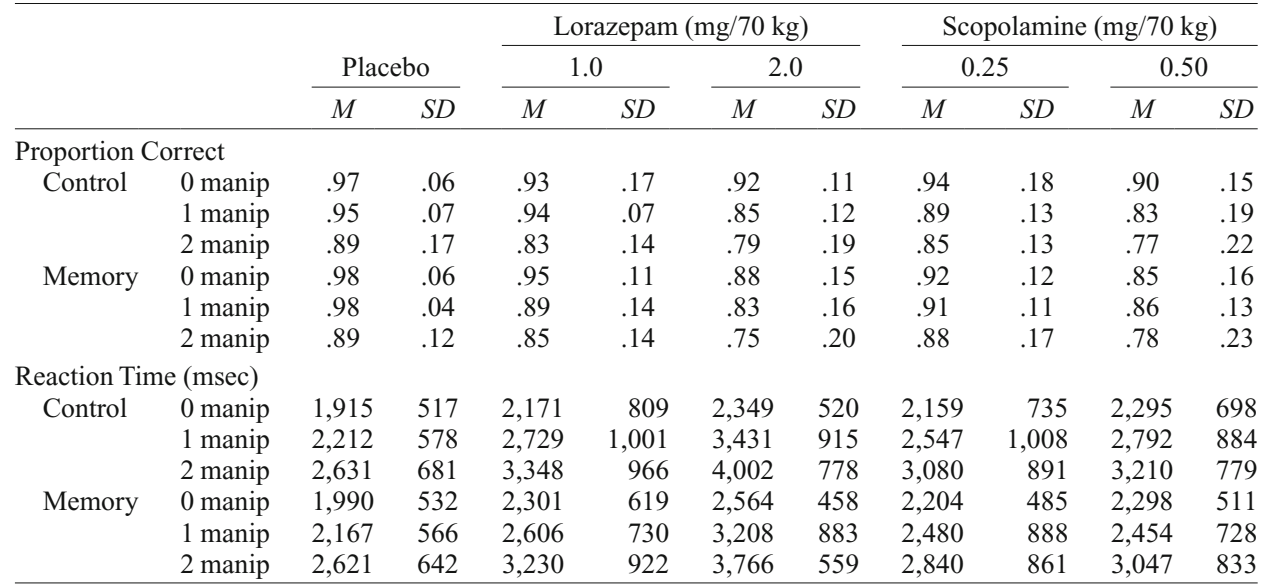

*Raw means and standard deviations are shown (excluding missing data). 

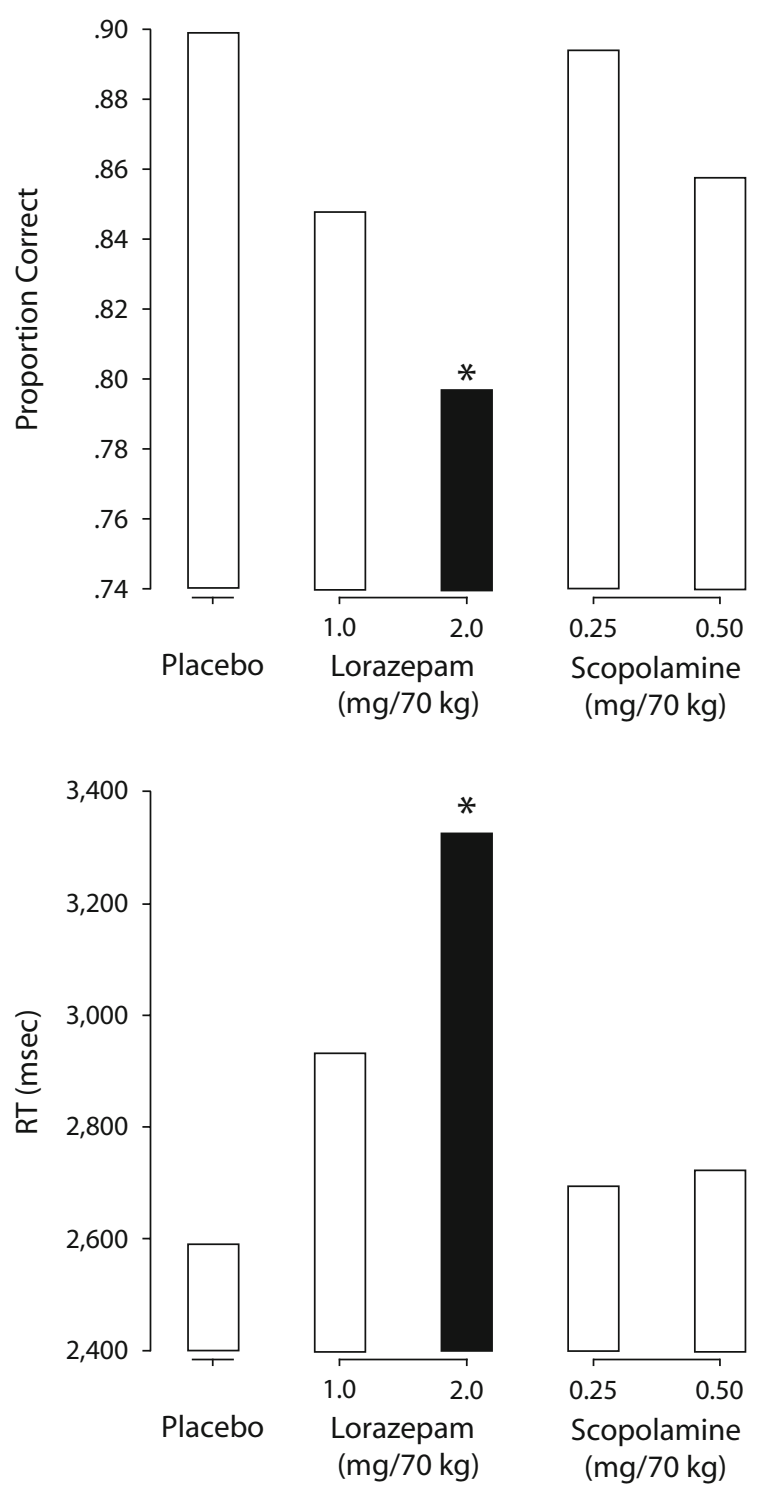

Figure 4. Mean proportion correct (top panel) and reaction time (bottom panel) for the 1- and 2-manipulation memory conditions of the manipulation task (adjusted means from the ANCOVA with the 0-manipulation memory condition as covariate; collapsed across number of manipulations and memory set size) as a function of drug condition. A darkened bar indicates an active drug value that is significantly different from placebo. An asterisk (*) indicates a lorazepam value that is significantly different from the scopolamine value at the corresponding dose.

lorazepam than for scopolamine when manipulation was required (Figure 4), suggesting that lorazepam produces relatively greater impairment in manipulation processes. There were no between-drug differences in effects of memory load (memory set size), suggesting similar effects on storage processes. In fact, drug effects in comparison with placebo did not increase as a function of memory set size, suggesting that the effect of memory load on working memory is not affected by these drugs, at least within the limited range (5 and 7 letters) tested in this study. The suggested absence of drug effects on storage capacity is consistent with the lack of effects of lorazepam and scopolamine in previous studies on span tasks, in which participants are asked to repeat random sequences of stimuli (e.g., digits) immediately after their presentation. Given that there is no delay between presentation and repetition, these tasks measure storage capacity (the maximum number of stimuli the participant is able to repeat), while minimizing rehearsal requirements.

Taken together, the accuracy results provide evidence for differential effects of scopolamine and lorazepam on working memory maintenance (rehearsal) versus manipulation processes. In addition to adding to the accumulating body of evidence supporting the distinction between maintenance and manipulation processes, these results suggest that the cholinergic system may be relatively more involved in maintenance processes, whereas the GABAergic system may be relatively more involved in manipulation processes. The suggestion that the cholinergic system is relatively more involved in maintenance processes is also supported by the finding that scopolamine but not diazepam produced delay-dependent impairment in a delayed matching-to-sample task in rhesus monkeys (Hironaka et al., 1992). Results of previous cognitive psychopharmacological studies in healthy volunteers also suggest different patterns of effects on working memory maintenance and manipulation as a function of neurochemical/ pharmacological mechanism of action. For example, the N-methyl-D-aspartic acid (NMDA) antagonist ketamine was shown to impair manipulation but not maintenance processes (Honey et al., 2003), and the nonamphetamine psychostimulant modafinil (whose mechanism of action remains unclear) was shown to enhance maintenance (at a long delay but not at short delays) as well as manipulation (Müller, Steffenhagen, Regenthal, \& Bublak, 2004). In comparison with placebo, manipulation was shown to be impaired by the centrally acting noradrenergic $\beta$-blocker propranolol but not by the peripherally acting $\beta$-blocker atenolol (Müller, Mottweiler, \& Bublak, 2005). Studies in which drugs with different mechanisms of action are directly compared at multiple doses, while working memory requirements are parametrically varied (as in the present study), are needed to further elucidate the neurochemical mechanisms underlying specific working memory processes. In addition, studies that combine cognitive psychopharmacological and neuroimaging techniques have the potential to identify the specific brain regions that are differentially affected by different drugs during the operation of specific working memory processes.

The RT data reveal a somewhat more complex pattern than the accuracy data. The pattern of results in the maintenance task does not provide evidence for relatively greater impairment for scopolamine than for lorazepam in rehearsal processes (as does the accuracy data), but rather seems to support greater overall slowing of working memory processes with lorazepam than with scopolamine (Figure 3), in that RT was longer for lorazepam than for scopolamine at corresponding dose levels. It is important to note that unlike the effect of scopolamine on accuracy, the effect of lorazepam on RT did not interact with delay in the maintenance task. This pattern 
suggests that the effect on RT does not reflect relatively greater impairment for lorazepam than for scopolamine in rehearsal processes but rather greater overall slowing with lorazepam than with scopolamine. The finding that RT was longer for high-dose lorazepam than for scopolamine when manipulation was required (Figure 4) does support the hypothesis (as does the accuracy data) that lorazepam produces relatively greater impairment in manipulation processes. Specifically, the finding that RT was longer for lorazepam than for scopolamine after effects on maintenance were controlled via ANCOVA suggests that in addition to producing an overall slowing of working memory processes (as suggested by the pattern of results in the maintenance task), lorazepam may produce additional slowing when manipulation is required. The finding of greater overall slowing with lorazepam than with scopolamine is consistent with the results of a previous study in our laboratory, in which RT in a nonmemory task (Stroop color-word) was longer with lorazepam than with scopolamine (Mintzer \& Griffiths, 2003). This finding is also consistent with results of previous studies in which RTs in other working memory tasks (a logical reasoning task designed to tap the central executive and a mental rotation task designed to measure visuospatial working memory) were longer with lorazepam or diazepam than with scopolamine (Bishop et al., 1996; Curran, Schifano, \& Lader, 1991; Rusted et al., 1991). However, to our knowledge, this is the first study to examine drug effects on working memory RT while controlling for drug effects on nonmemory RT in the same task, and to examine drug effects on working memory manipulation RT while controlling for drug effects on working memory maintenance RT. This design enables the conclusion to be drawn that in comparison with scopolamine, lorazepam produces greater overall slowing of working memory processes as well as of manipulation processes specifically.

One potential limitation of the manipulation task is that only the memory trials were included in the analyses. As described in the Results, data from the control trials were excluded after it became clear from the pattern of results that the control condition did not serve as a nonmemory control for the 1- and 2-manipulation conditions, in which the participant must still maintain the reordered string in working memory. However, an ANCOVA was conducted on data from the memory trials, with the 0 -manipulation condition as covariate, allowing conclusions to be drawn about drug effects on manipulation while controlling for effects on maintenance and other processes that are common to all three manipulation conditions (e.g., attention, psychomotor coordination). Although the 0 -manipulation condition cannot control for drug effects specifically on alphabetization, to our knowledge, there is no evidence of differences between lorazepam and scopolamine in the cognitive processes that underlie alphabetization.

In summary, using an experimental design in which both drug dose and processing requirements were parametrically manipulated while drug effects on nonmemory processes were controlled for, the present study provided evidence for differential effects of lorazepam and sco- polamine on working memory maintenance (rehearsal) versus manipulation processes, and for greater overall slowing of working memory processes with lorazepam. In addition to supporting the distinction between maintenance and manipulation processes, the results enhance the understanding of the neurochemical mechanisms underlying these working memory processes.

\section{AUTHOR NOTE}

This project was supported by National Institute on Drug Abuse Research Grants DA-03889 and DA-11936. The authors thank Jeanene Pope and Koty Nadeau for protocol management and technical assistance, John Yingling for computer programming assistance and technical support, and Paul Nuzzo for assistance with data analysis. Correspondence concerning this article should be addressed to M. Z. Mintzer, Department of Psychiatry and Behavioral Sciences, Behavioral Biology Research Center, Johns Hopkins University School of Medicine, 5510 Nathan Shock Drive, Baltimore, MD 21224 (e-mail: mmintzer@jhmi.edu).

\section{REFERENCES}

Atkinson, R. C., \& Shiffrin, R. M. (1968). Human memory: A proposed system and its control processes. In K. W. Spence \& J. T. Spence (Eds.), The psychology of learning and motivation: Advances in research and theory (Vol. 2, pp. 89-195). New York: Academic Press.

BADDELEY, A. [D.] (1986). Working memory. Oxford: Oxford University Press, Clarendon Press.

BADDELEY, A. D. (1992). Working memory: The interface between memory and cognition. Journal of Cognitive Neuroscience, 4, 281-288.

BARCH, D. M. (2004). Pharmacological manipulation of human working memory. Psychopharmacology, 174, 126-135.

BARDE, L. H., \& THOMPSON-SCHILL, S. L. (2002). Models of functional organization of the lateral prefrontal cortex in verbal working memory: Evidence in favor of the process model. Journal of Cognitive Neuroscience, 14, 1054-1063.

Berman, K. F., Austin-Lane, J. L., Esposito, G., Van Horn, J. D., \& Weinberger, D. R. (1996). Dissecting the "working" and the "memory" in a PET study of working memory using graded tasks and isomorphic stimuli. NeuroImage, 3, S529.

Bishop, K. I., Curran, H. V., \& Lader, M. (1996). Do scopolamine and lorazepam have dissociable effects on human memory systems? A dose-response study with normal volunteers. Experimental \& Clinical Psychopharmacology, 4, 292-299.

CHROBAK, J. J., \& NAPIER, T. C. (1992). Delayed-non-match-to-sample performance in the radial arm maze: Effects of dopaminergic and gabaergic agents. Psychopharmacology, 108, 72-78.

Coull, J. T., Middleton, H. C., Robbins, T. W., \& Sahakian, B. J. (1995). Contrasting effects of clonidine and diazepam on tests of working memory and planning. Psychopharmacology, 120, 311-321.

Curran, H. V. (1991). Benzodiazepines, memory, and mood: A review. Psychopharmacology, 105, 1-8.

CURRan, H. V. (2000). Psychopharmacological approaches to human memory. In M. S. Gazzaniga (Ed.), The new cognitive neurosciences (2nd ed., pp. 797-804). Cambridge, MA: MIT Press.

Curran, H. V., Schifano, F., \& Lader, M. (1991). Models of memory dysfunction? A comparison of the effects of scopolamine and lorazepam on memory, psychomotor performance and mood. Psychopharmacology, 103, 83-90.

D'EsPosito, M. (2001). Functional neuroimaging of working memory. In R. Cabeza \& A. Kingstone (Eds.), Handbook of functional neuroimaging of cognition (pp. 293-327). Cambridge, MA: MIT Press.

D’Esposito, M., \& Postle, B. R. (1999). The dependence of span and delayed-response performance on prefrontal cortex. Neuropsychologia, 37, 1303-1315.

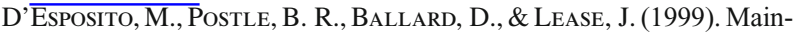
tenance versus manipulation of information held in working memory: An event-related fMRI study. Brain \& Cognition, 41, 66-86.

D’Esposito, M., Postle, B. R., \& RYPMA, B. (2000). Prefrontal cortical 
contributions to working memory: Evidence from event-related fMRI studies. Experimental Brain Research, 133, 3-11.

Duka, T., Curran, H. V., Rusted, J. M., \& Weingartner, H. J. (1996). Perspectives on cognitive psychopharmacology research. Behavioural Pharmacology, 7, 401-410.

Furey, M. L., Pietrini, P., Alexander, G. E., Schapiro, M. B., \& Horwitz, B. (2000). Cholinergic enhancement improves performance on working memory by modulating the functional activity in distinct brain regions: A positron emission tomography regional cerebral blood flow study in healthy humans. Brain Research Bulletin, 51, 213-218.

Furey, M. L., Pietrini, P., \& Haxby, J. V. (2000). Cholinergic enhancement and increased selectivity of perceptual processing during working memory. Science, 290, 2315-2319.

Furey, M. L., Pietrini, P., Haxby, J. V., Alexander, G. E., Lee, H. C., VANMETER, J., ET AL. (1997). Cholinergic stimulation alters performance and task-specific regional cerebral blood flow during working memory. Proceedings of the National Academy of Sciences, 94, 6512-6516.

Glahn, D. C., Kim, J., Cohen, M. S., Poutanen, V. P., Therman, S., BAVA, S., ET AL. (2002). Maintenance and manipulation in spatial working memory: Dissociations in the prefrontal cortex. NeuroImage, 17, 201-213.

Glasky, A. J., Melchior, C. L., Pirzadeh, B., Heydari, N., \& RitZMANN, R. F. (1994). Effect of AIT-082, a purine analog, on working memory in normal and aged mice. Pharmacology Biochemistry \& Behavior, 47, 325-329.

HiRONAKA, N., MiYATA, H., \& ANDO, K. (1992). Effects of psychoactive drugs on short-term memory in rats and rhesus monkeys. Japanese Journal of Pharmacology, 59, 113-120.

Hirshman, E., Fisher, J., Henthorn, T., ARndt, J., \& Passannante, A. (2002). Midazolam amnesia and dual process models of the word frequency mirror effect. Journal of Memory \& Language, 47, 499-516.

Hirshman, E., Passannante, A., \& Arndt, J. (2001). Midazolam amnesia and conceptual processing in implicit memory. Journal of Experimental Psychology: General, 130, 453-465.

Honey, R. A., Turner, D. C., Honey, G. D., Sharar, S. R., Kumaran, D., Pomarol-Clotet, E., ET Al. (2003). Subdissociative dose ketamine produces a deficit in manipulation but not maintenance of the contents of working memory. Neuropsychopharmacology, 28, 2037-2044.

Ingles, J. L., Beninger, R. J., Jhamandas, K., \& Boegman, R. J. (1993). Scopolamine injected into the rat amygdala impairs working memory in the double Y-maze. Brain Research Bulletin, 32, 339-344.

KePPEL, G. (1991). Design and analysis: A researcher's handbook (3rd ed.). Englewood Cliffs, NJ: Prentice Hall.

Ketchum, J. S., Sidell, F. R., Crowell, E. G., Aghajanian, G. K., \& HaInes, A. H. (1973). Atropine, scopolamine, and ditran: Comparative pharmacology and antagonists in man. Psychopharmacologia, 28, 121-145.

Kim, J., Glahn, D. C., Nuechterlein, K. H., \& Cannon, T. D. (2004). Maintenance and manipulation of information in schizophrenia: Further evidence for impairment in the central executive component of working memory. Schizophrenia Research, 68, 173-187.

Kopelman, M. D. (1986). The cholinergic neurotransmitter system in human memory and dementia: A review. Quarterly Journal of Experimental Psychology, 38A, 535-573.

Levy, A., Brandeis, R., Treves, T. A., Meshulam, Y., Mawassi, F., FEILER, D., ET AL. (1994). Transdermal physostigmine in the treatment of Alzheimer's disease. Alzheimer Disease \& Associated Disorders, $\mathbf{8}, 15-21$.

Miller, G. A. (1956). The magical number seven, plus or minus two: Some limits on our capacity for processing information. Psychological Review, 63, 81-97.

MinTZER, M. Z. (2003). Triazolam-induced amnesia and the wordfrequency effect in recognition memory: Support for a dual process account. Journal of Memory \& Language, 48, 596-602.

Mintzer, M. Z., Frey, J. M., Yingling, J. E., \& Griffiths, R. R. (1997). Triazolam and zolpidem: A comparison of their psychomotor, cognitive, and subjective effects in healthy volunteers. Behavioural Pharmacology, 8, 561-574.

MinTZER, M. Z., \& GRIFFITHS, R. R. (2001a). Acute dose-effects of scopolamine on false recognition. Psychopharmacology, 153, 425-433.
Mintzer, M. Z., \& GRiffiths, R. R. (2001b). False recognition in triazolam-induced amnesia. Journal of Memory \& Language, 44, 475-492.

MinTZER, M. Z., \& GRIFFiths, R. R. (2003). Lorazepam and scopolamine: A single-dose comparison of effects on human memory and attentional processes. Experimental \& Clinical Psychopharmacology, 11, 56-72.

MOHLER, H., \& OKada, T. (1977). Benzodiazepine receptor: Demonstration in the central nervous system. Science, 198, 849-851.

Müller, U., Mottweiler, E., \& Bublak, P. (2005). Noradrenergic blockade and numeric working memory in humans. Journal of Psychopharmacology, 19, 21-28.

Müller, U., Steffenhagen, N., Regenthal, R., \& Bublak, P. (2004). Effects of modafinil on working memory processes in humans. Psychopharmacology, 177, 161-169.

Petrides, M. (1994). Frontal lobes and behavior. Current Opinion in Neurobiology, 4, 207-211.

PoLSTER, M. R. (1993). Drug-induced amnesia: Implications for cognitive neuropsychological investigations of memory. Psychological Review, 114, 477-493.

Postle, B. R., Berger, J. S., \& D’Esposito, M. (1999). Functional neuroanatomical double dissociation of mnemonic and executive control processes contributing to working memory performance. Psychology, 96, 12959-12964.

Reder,L.M., OAtes, J. M.,Thornton,E. R., Quinlan,J. J., Kaufer, A., \& SAUER, J. (2006). Drug-induced amnesia hurts recognition, but only for memories that can be unitized. Psychological Science, 17, 562-567.

Robbins, T. W., Semple, J., Kumar, R., Truman, M. I., Shorter, J., Ferraro, A., ET AL. (1997). Effects of scopolamine on delayedmatching-to-sample and paired associate's tests of visual memory and learning in human subjects: Comparison with diazepam and implications for dementia. Psychopharmacology, 134, 95-106.

Rusted, J. M. (1988). Dissociative effects of scopolamine on working memory in healthy young volunteers. Psychopharmacology, 96, 487-492.

Rusted, J. M., Eaton-Williams, P., \& Warburton, D. M. (1991). A comparison of the effects of scopolamine and diazepam on working memory. Psychopharmacology, 105, 442-445.

Rusted, J. M., \& WARBURTON, D. M. (1988). The effects of scopolamine on working memory in healthy young volunteers. Psychopharmacology, 96, 145-152.

SaNo, M., Bell, K., Marder, K., Stricks, L., Stern, Y., \& Mayeux, R. (1993). Safety and efficacy of oral physostigmine in the treatment of Alzheimer disease. Clinical Neuropharmacology, 16, 61-69.

SMITH, E. E., \& JonIDES, J. (1998). Neuroimaging analyses of human working memory. Proceedings of the National Academy of Sciences, 95, 12061-12068.

SQUIRES, R. F., \& BRAESTRUP, C. (1977). Benzodiazepine receptors in rat brain. Nature, 266, 732-734.

SternberG, S. (1969). The discovery of processing stages: Extensions of Donder's method. Acta Psychologica, 30, 276-315.

Terry, A. V., Jr., Jackson, W. J., \& BuCCAFUSCO, J. J. (1993). Effects of concomitant cholinergic and adrenergic stimulation on learning and memory performance by young and aged monkeys. Cerebral Cortex, 3, 304-312.

WeCHSLER, D. (1981). Manual for the Wechsler Adult Intelligence Scale, revised. New York: Psychological Corporation.

Wesnes, K., Simpson, P., \& Kidd, A. (1988). An investigation of the range of cognitive impairments induced by scopolamine $0.6 \mathrm{mg}$ s.c. Human Psychopharmacology, 3, 27-41.

White, A. M., Simson, P. E., \& Best, P. J. (1997). Comparison between the effects of ethanol and diazepam on spatial working memory in the rat. Psychopharmacology, 133, 256-261.

WiLliAMS, E. J. (1949). Experimental designs balanced for the estimation of residual effects of treatments. Australian Journal of Scientific Research, 2, 149-168.

(Manuscript received May 3, 2006; revision accepted for publication December 7, 2006.) 